\title{
Die wahrgenommene feministische Bedrohung: Empirische Befunde zum Antifeminismus in Deutschland
}

\author{
Annette Schnabel · Heiko Beyer • Bettina Ülpenich
}

Online publiziert: 6. Dezember 2021

(C) Der/die Autor(en) 2021

Zusammenfassung Die Frauenbewegung war von Beginn an von antifeministischen Diskursen und Initiativen begleitet. Diese reagieren auf den Erfolg feministischer Bewegung und Politik. Mit einer empirischen Analyse antifeministischer Einstellungen auf Grundlage einer quantitativen Bevölkerungsbefragung können wir zeigen, dass in Deutschland aktuelle antifeministische Überzeugungen nicht nur in ein umfassendes Einstellungscluster eingebettet sind, das eine konservativ-traditionelle Geschlechterordnung widerspiegelt, sondern sich auch als Reaktion auf als negativ empfundene Folgen des institutionellen und gesamtgesellschaftlichen Wandels verstehen lassen müssen.

Schlüsselwörter Antifeminismus $\cdot$ Misogynie $\cdot$ Sexismus $\cdot$ Feminismus $\cdot$ Soziale Mobilisierung

\footnotetext{
Annette Schnabel $(\varangle) \cdot$ Heiko Beyer $\cdot$ Bettina Ülpenich Institut für Sozialwissenschaften, Abteilung Soziologie, Heinrich-Heine-Universität Düsseldorf, Universitätsstr. 1, 40225 Düsseldorf (Postadresse), Deutschland

E-Mail: schnabel@hhu.de

Heiko Beyer

E-Mail: Heiko.Beyer@uni-duesseldorf.de

Bettina Ülpenich

E-Mail: Bettina.Uelpenich@uni-duesseldorf.de
} 


\title{
"Feminists disturb the social order"-Empirical findings on antifeminism in Germany
}

\begin{abstract}
The women's movement has been accompanied by antifeminist discourses and initiatives from its beginning. Those can be considered a response to the success of feminist movements and politics. Using quantitative survey data of antifeminist attitudes, we show that recent antifeminist beliefs in Germany are not only embedded in a comprehensive attitudinal cluster of a traditional-conservative gender order but can also be understood as a reaction towards negatively perceived consequences of institutional and social change.
\end{abstract}

Keywords Antifeminism $\cdot$ Misogyny $\cdot$ Sexism $\cdot$ Feminism $\cdot$ Social mobilisation

\section{Einführung}

Die Frauenbewegung gehört mit ihren vielfältigen Formen und Anliegen zu den ältesten sozialen Bewegungen der Moderne und ist vielleicht eine ihrer erfolgreichsten. ${ }^{1}$ Sie ist angetreten, die traditionelle Geschlechterordnung zu verändern, die lange Zeit die Exklusion von Frauen aus vielen gesellschaftlichen Bereichen wie Erwerbsarbeit, politischer Mitbestimmung, universitärer Wissensproduktion und männliche Übergriffe auf den weiblichen Körper als selbstverständlich legitimierte. Die Frauenbewegung wurde dabei seit ihren Anfängen von antifeministischen Protesten begleitet, die sich gegen den angestrebten sozialen Wandel richteten.

Auch aktuell finden sich Feminist*innen immer wieder mit antifeministischen, sexistischen und anti-genderistischen Angriffen konfrontiert. Diese äußern sich in Statements von Politiker*innen, in den Kommentarspalten von Online-Medien, in Tweets und \#-Kampagnen ${ }^{2}$ und in persönlichen, alltäglichen Interaktionen. Sie lassen sich als öffentlicher Ausdruck mobilisierter individueller antifeministischer Einstellungen verstehen.

Der vorliegende Beitrag widmet sich dem Phänomen des Antifeminismus in Deutschland und beleuchtet seine Verbreitung und kognitive Einbettung in weiter gefasste Weltanschauungssysteme. Forschungsleitend sind die beiden Fragen, wie verbreitet antifeministische Einstellungen sind und mit welchen anderen Einstellungen und Weltinterpretationen sie verknüpft sind.

Dazu werden im ersten Teil des Artikels theoretische Überlegungen zur Gegenmobilisierung von feministischen Bewegungen (Abschnitt 1) sowie bisherige Erkenntnisse zum Antifeminismus in Deutschland (Abschnitt 2) referiert. Im dritten Abschnitt werden weltanschauliche Einbettungsprozesse des Antifeminismus disku-

\footnotetext{
1 Der hier verwendete Singular soll nicht über die Verschiedenheiten feministischer Anliegen, Aktionsformen und Organisationen hinwegtäuschen. Eine ausführliche Begründung für unsere Entscheidung, dennoch den Singular zu verwenden, findet sich in Abschnitt 1.

2 Vgl. die Kampagne „Why I am not feminist“: https://www.theodysseyonline.com/25-reasons-why-notfeminist.
} 
tiert und im vierten eigene Daten vorgestellt, die auf einer standardisierten OnlineBefragung aus dem Jahr $2016(n=1202)$ basieren und vorliegende qualitative Arbeiten zum Antifeminismus ergänzen sollen. Unsere Untersuchung zeigt, mit welchen personenbezogenen Merkmalen antifeministische Einstellungen zusammenhängen und mit welchen Weltanschauungsbestandteilen sie verknüpft sind.

\section{Gegenmobilisierung und Feminismus}

Soziale Bewegungen, denen es gelungen ist, ihre Anliegen öffentlich sichtbar zu machen, müssen in der Regel mit Gegenmobilisierung rechnen. Dies gilt auch für feministische Anliegen. Ob Antifeminismus bereits als eigenständige soziale Bewegung bezeichnet werden kann, ist eine offene Frage (Kaiser 2020, S. 79 ff.), dennoch lassen sich die damit verbundenen Einstellungen, politischen Positionen und weltanschaulichen Perspektiven am ehesten verstehen, wenn sie im Rahmen der Erkenntnisse der Bewegungsforschung analysiert werden, befasst sich diese doch mit dem Wer, Was und Warum der sozialen Mobilisierung von Einstellungen und daraus resultierenden Protesthandlungen. Im Folgenden sollen einem Artikel angemessen kurz bewegungssoziologische Erkenntnisse zu Gegenmobilisierung, feministischen Bewegungen und Antifeminismus vorgestellt werden, um anschließend die empirischen Erkenntnisse zu antifeministischen Einstellungen zu kontextualisieren.

Verglichen mit der wissenschaftlichen Beschäftigung mit sozialen Bewegungen ist die Auseinandersetzung mit Gegenmobilisierung derzeit noch wenig ausgeprägt. Im Rahmen des ,dynamics of contention“-Ansatzes (McAdam et al. 2001) geraten allerdings in jüngerer Zeit vor allem konservative Parteien, Organisationen und Bewegungen mehr und mehr als Verteidiger des Status quo in den Blick (z. B. Krause et al. 2015; Hawley 2017; Reuband 2017). ${ }^{3}$

Gegenmobilisierung richtet sich gegen durch soziale Bewegungen (vermeintlich) erfolgreich angestoßenen sozialen Wandel: Während soziale Bewegungen Gruppen, die in der gesellschaftlichen Hierarchie höher stehen, herausfordern, richtet sich Gegenmobilisierung gegen solche Veränderungsbestrebungen ,,von unten“ (Mottl 1980, S. 621). Allerdings ist nicht jede Gegenmobilisierung schon eine Gegenbewegung. Gegenmobilisierung findet statt, wenn individuelle Einstellungen öffentlich - z. B. über soziale Medien - sichtbar, geteilt und artikulierbar werden. Ob daraus eine Bewegung mit einem organisierten Kern werden kann, entscheidet sich in Abhängigkeit verschiedener individueller und gesellschaftlicher Faktoren. ${ }^{4}$ Bisherige Untersuchungen zeigen, dass Gegenmobilisierungen jenseits ihres Organisationsgrades bestimmte strukturelle Eigenheiten aufweisen und dass ihre Interessent*innen einige einstellungsrelevante Eigenschaften teilen.

\footnotetext{
3 Die Thematisierung konservativer Gegenmobilisierung findet überwiegend im Rahmen der Auseinandersetzung um die Neue Rechte und um Nationalismus statt, weniger im Rahmen der Erforschung sozialer Bewegungen. Dies liegt vermutlich daran, dass die Bewegungsforschung ihren Blick zumeist auf Bewegungen richtet, die den Staat herausfordern (Meyer und Staggenborg 1996, S. 1629). Seit rechte und nationalistische Bewegungen verstärkt als „Gefahr für die Demokratie“ angesehen werden, rücken auch sie stärker in den Fokus der Bewegungsforschung.

4 Vgl. hierzu u. a. Meyer und Staggenborg (1996) und McAdam et al. (2001).
} 
Mottl argumentiert, dass Gegenmobilisierung ein bewusster Versuch sei ,to resist or to reverse social change“ (Mottl 1980, S. 620). Hinsichtlich der Mobilisierungsgründe kommt Tilly (1976; [1964]) zu dem Ergebnis, dass zu schnelles Wachstum im Zuge sozialen Wandels und die damit einhergehende Umverteilung von Ressourcen einen solchen Widerstand hervorrufen könnten. Inwieweit dies auch für die Mobilisierung antifeministischer Einstellungen gilt, ist eine noch zu klärende Frage.

Über die soziodemografische Verortung von Gegenmobilisierung gibt es unterschiedliche Hypothesen: Während Tilly feststellt, dass sich nicht nur Verlierer gegen sozialen Wandel wenden, konstatiert Mottl (1980, S. 621 [Herv. d. Autor.]), Gegenmobilisierung sei ,related to social divisions resulting from socioeconomic decline, threatening the position of those who mobilise". Auch dies wird für die Mobilisierung von antifeministischen Einstellungen zu untersuchen sein.

Jenseits der Fragen nach Gründen und Merkmalen der Protagonist*innen weist die Bewegungsforschung auf die kontextuelle Einbettung von Gegenmobilisierung hin: Gegenmobilisierung findet im Dreiecksverhältnis zwischen sozialer Bewegung und staatlichen Policies statt (Mottl 1980; Rucht 1991; Meyer und Staggenborg 1996): Gegenmobilisierung ist unwahrscheinlich, wenn soziale Bewegungen ein politisches Feld nicht erfolgreich besetzen können oder wenn die durch die soziale Bewegung angestoßenen Policy-Änderungen als irreversibel wahrgenommen werden, da es dann an einer für die Gegenmobilisierung vorteilhaften ,political opportunity structure“ (Eisinger 1973, S. 11) fehlt. Verbreitung und Sichtbarkeit von Gegenmobilisierung hängt zudem von ihrer ideologischen Einbettung ab: Mottl (1980, S. 622) sowie Meyer und Staggenborg (1996, S. 1639) stellen hierzu fest, dass Gegenmobilisierungen erfolgreicher sind, wenn ihre Anliegen entlang eines aktuellen Wertekonflikts positioniert sind.

Antifeministische Gegenmobilisierung bezieht sich konkret auf Feminismen als gesellschaftsverändernde Kraft. Antifeminismus spiegelt sich in allen öffentlich beobachtbaren Statements gegen vermeintliche und reale feministische Erfolge, in entsprechenden sozialen Normierungen und Praktiken und realisiert sich, wie später zu zeigen sein wird, in individuellen Einstellungen, auch wenn viele Menschen, die solchermaßen antifeministische Einstellungen teilen, diesen Bezugspunkt nur vage umreißen können. Für antifeministische Weltbilder und Handlungskonsequenzen ist es jedoch unerheblich, ob antifeministische Wahrnehmungen feministische Anliegen angemessen oder ,richtig“ reflektieren, da es bereits hinreicht, dass diese Wahrnehmungen subjektiv relevant und sozial geteilt sind, um ,real in their consequences“ (Thomas und Thomas 1928) zu sein. ${ }^{5}$

Feministische Anliegen, gegen die sich auch der moderne Antifeminismus richtet, werden seit den 1840er-Jahren in vielfältiger Weise vertreten: Es haben sich verschiedene Wellen und Strömungen ausdifferenziert, die teils sogar einander widersprechende Zielsetzungen verfolgen, ${ }^{6}$ sodass umstritten ist, ob überhaupt von einer einheitlichen Bewegung gesprochen werden kann. Aus Sicht der Bewegungs-

\footnotetext{
5 Wie weit feministische Anliegen und ihre antifeministische Wahrnehmung auseinanderfallen und wie konsequenzenreich diese Wahrnehmung sein kann, zeigt Kaiser (2020) in erschreckender Eindringlichkeit.

${ }^{6}$ Für einen umfassenden Überblick über die Geschichte der deutschen Frauenbewegung vgl. u. a. NaveHerz (1997); Gerhard (1996).
} 
forschung ist dies nicht überraschend, da es sich um ein allgemeines epistemologisches Problem handelt, das sowohl Mitglieder einer sozialen Bewegung als auch Bewegungsforscher*innen gleichermaßen haben: In sozialen Bewegungen werden üblicherweise Situationsdefinitionen, Ziele und Strategien beständig neu verhandelt und dadurch wird die Einheitlichkeit des Phänomens infrage gestellt.

Dies kann nicht bedeuten, dass gänzlich auf die begriffliche Synthese verzichtet werden müsste, auch wenn mit einer solchen Suche nach dem gemeinsamen Nenner die Dynamiken der feministischen Anliegen zunächst gewissermaßen stillgestellt werden. Mit diesen Einschränkungen kann Feminismus analytisch als Weltdeutung und soziale Bewegung umrissen werden, ,die alle Bereiche des Menschlichen betrifft und den patriarchalen Gehalt aller kulturellen Hervorbringungen des Mannes (der sich traditionell als Mensch schlechthin definiert) bloßlegt und kritisiert" (Pusch 1983, S. 14). Dabei steht die Analyse der gesamtgesellschaftlichen Verhältnisse, also nicht nur der Beziehungen zwischen Frauen und Männern, im Vordergrund: „Das Ziel des Feminismus ist [...] die gesellschaftliche Behandlung des Frau-Seins zu verbessern, unter der einzelne Frauen heute als Frauen leiden“ (Radcliffe Richards 1983, S. 20). Diese „transformative Politik“ ist „darauf gerichtet, gesellschaftliche Institutionen zu verändern, jede Form von Unterdrückung zu überwinden“ (List 1989, S. 10). Damit einher geht die explizite feministische Forderung, die gültige Geschlechterordnung infrage zu stellen, welche sich nicht allein in die öffentliche Sphäre der Erwerbsarbeit oder politischen Mitbestimmung erstreckt, sondern auch in die Privatsphäre der Familie und Partnerschaft.

\section{Forschungsstand zum Antifeminismus}

Antifeminismus richtet sich explizit gegen alle Formen von Feminismus und gegen die politischen und sozialen Errungenschaften der Geschlechtergerechtigkeit (Schmincke 2018, S. 28; Herrmann 2015, S. 79). Antifeminismus ist jedoch nicht gleichzusetzen mit Frauenfeindlichkeit oder Sexismus, da beides historisch lange vor dem Antifeminismus entstanden ist (Schenk 1981, S. 163). Des Weiteren ist der Begriff des Antifeminismus von jenem des „Anti-Genderismus“ zu unterscheiden. Letzterer bezeichnet laut Hark und Villa (2015a, S. 20 ff.) die Ablehnung der wissenschaftlichen und politischen Infragestellung des alltagsweltlichen Verständnisses von Zweigeschlechtlichkeit. Anti-Genderismus kann Bestandteil antifeministischer Überzeugungen sein (Schmincke 2018, S. 28), muss dies aber nicht, wie das Beispiel der sogenannten TERFS (trans-exclusionary radical feminists) zeigt (Williams 2016).

Chafetz und Dworkin legten 1987 eine der ersten wissenschaftlich-theoretischen Analysen zu Antifeminismus vor. Sie konnten zeigen, dass feministische Anliegen seit Beginn der Frauenbewegung Mitte des 19. Jahrhunderts antifeministische Reaktionen wie Anti-Suffragette-, Pro-life- oder Väter-Bewegungen auslösten. Chafetz und Dworkin (1987, S. 35) folgern aus der anti-institutionellen Haltung feministischer Bewegungen, dass Antifeminismus ,proinstitutional in character" sei. Als potenzielle Akteur*innen machen sie Gruppen mit starken Interessen (,,vested interest groups") wie z.B. Vertreter*innen der Kirchen, aber auch Frauen aus, die 
befürchteten, durch die Öffnung der öffentlichen Sphäre für Frauen an Ansehen als „Managerinnen“ des Häuslichen zu verlieren, sei es, weil der Status ihrer Ehemänner oder ihr eigener Hausfrauenstatus einer Abwertung unterworfen sein könnte. Die damit verbundenen antifeministischen Einstellungen seien jedoch in bekannte Einstellungsmuster eingebettet: „A conspicuous backlash does not reflect a significant change in public attitudes, but a coalescence of diffuse opposition that was always present" (Chafetz und Dworkin 1987, S. 42). Antifeminismus erscheine in Bezug auf seine Anliegen und Sympathisant*innen weit weniger ausdifferenziert als die heterogenen und variantenreichen feministischen Strömungen: Die meisten der von den Autor*innen untersuchten antifeministischen Initiativen leiteten ihre Legitimation aus einem traditionellen, essentialistisch begründeten Geschlechterbild ab, das Männern die Rolle des Familienoberhaupts und Ernährers, Frauen jene der, für reproduktive Haushaltstätigkeiten prädestinierten, Mutter zuweisen (Chafetz und Dworkin 1987, S. 42 f.). ${ }^{7}$

Im Gegensatz zum Feminismus ist Antifeminismus weniger systematisch wissenschaftlich erschlossen, aber die Zahl der Studien wächst auch in Deutschland kontinuierlich. Historische Untersuchungen zum deutschen Antifeminismus konzentrieren sich bisher vor allem auf das Kaiserreich: die Wende vom 19. zum 20. Jahrhundert ist die Periode, in der naturwissenschaftlich legitimierte Erklärungsgrundlagen für misogyne Perspektiven ${ }^{8}$ immer stärker an Relevanz gewinnen (Amlinger 2015, S. 2). Eine der wichtigsten Typologien antifeministischer Ideologien aus dieser Zeit stammt von Hedwig Dohm (1902), ${ }^{9}$ die zeigt, wie ausdifferenziert das Feld des Antifeminismus damals schon gewesen ist, und dass häufig auch Frauen entsprechende Positionen vertraten.

Für Volkov (1978) ist die Wilhelminische Zeit durch einen starken antimodernen Impuls geprägt, der sich in überzogenem Nationalismus, imperialen Bestrebungen, Kriegsbegeisterung, aber auch einer konservativen Perspektive auf die Familie ausdrücke. Antifeminismus trete häufig gemeinsam mit antisemitischen Ressentiments auf, die sich ebenfalls gegen eine als bedrohlich wahrgenommene Moderne richteten. Antisemitismus sei dabei zu einem Kürzel für ein ganzes Einstellungssyndrom geronnen: „Women, like Jews, ran the argument, lacked the required ethical consciousness and moral vigour characteristic of German men. Antisemitism and antifeminism were almost invariably combined in Imperial Germany. Both were integral elements of the anti-emancipatory culture of a majority of Germans in the pre-war years“" (Volkov 1978, S. 34). ${ }^{10}$

\footnotetext{
7 Als „Klassiker“ der Antifeminismus-Analyse gilt das 1991 erschienene Buch Backlash. The Undeclared War Against American Women von Susan Faludi (vgl. Schmincke 2018, S. 30). Faludi kommt hier zu ähnlichen Ergebnissen wie Chafetz und Dworkin.

${ }^{8}$ Damit sind Positionen zusammengefasst, die ein essentialistisches Verständnis von Weiblichkeit und damit verbunden eine ontologische Vorstellung von der Minderwertigkeit von Frauen vertreten (Amlinger 2015, S. 2).

9 Vgl. Amlinger (2015, S. 3).

10 Peetz (2014) kann allerdings zeigen, dass zu dieser Zeit feministische Positionen nicht automatisch vor Antisemitismus schützten - dies lässt sich unter anderem am Antisemitismus der proletarisch-sozialistischen Frauenbewegung zeigen (Braukmann 2003).
} 
Planert (1998) wiederum kann belegen, dass Antifeminismus im Kaiserreich eine gut vernetzte Gegenbewegung war, die vor allem als Folge der gesellschaftlichen Infragestellung etablierter Geschlechterverhältnisse durch feministische Forderungen nach politischer, ökonomischer und sozialer Teilhabe gelesen werden könne.

Die meisten Erkenntnisse zum aktuellen Antifeminismus basieren auf qualitativen Untersuchungen. Darunter finden sich in jüngerer Zeit Analysen, die sich mit der Modernisierung antifeministischer Positionen in der Gegenwart beschäftigen (Hark und Villa 2015a; Lang und Peters 2018; Schmincke 2018), oder organisierte Formen des Antifeminismus analysieren (Rosenbrock 2012; Kemper 2012). Andere Studien untersuchen das Thema Antifeminismus diskurs- und inhaltsanalytisch in den Printmedien (Möller 1999) oder im Internet (Drüeke und Klaus 2014; Drüeke und Peil 2015; Drüeke und Zobl 2016).

Als Kampffelder der aktuellen antifeministischen Debatten werden insbesondere familienbezogene Themen identifiziert: Das durch die Emanzipation von Frauen vermeintlich gefährdete Kindswohl, die rechtliche Benachteiligung von Scheidungsvätern und das Bild männlicher Bildungsverlierer werden in der Literatur als wichtige Topoi genannt (Klaus 2008, S. 177ff.; Lang und Peters 2018; Schmincke 2018, S. 30). Mit Bezug auf die Präsenz von Antifeminismus in Internetportalen stellt Drüeke (2016, S. 9f.) fest, dass die Betonung traditioneller Geschlechterbilder und biologischer Erklärungsansätze den Kern der Auseinandersetzungen darstellen. Darüber hinaus finden sich Hinweise auf eine Verschränkung mit homophoben und nationalistischen Diskursen in den Internetportalen (Gesterkamp 2010; Rosenbrock 2012; Kemper 2011).

Organisatorisch spielen vor allem die seit den 1980er-Jahren etablierten Männergruppen eine große Rolle (Rosenbrock 2012). Das antifeministische Themenspektrum scheint eng verbunden mit der Überzeugung, dass die rechtliche Gleichheit von Männern und Frauen längst hergestellt sei und weitere Gleichstellungsansprüche von Frauen unweigerlich zur Benachteiligung von Männern führen. Damit wird der Kampf gegen staatliche Gleichstellungs- und Bildungspolitiken begründet (Rosenbrock 2012; Lang und Peters 2018, S. 20; Laumann und Debus 2018). Legitimiert wird die gesellschaftliche (Zurück-)Verweisung von Frauen in die häusliche Reproduktionssphäre über die kategorische Ablehnung aller (sozialwissenschaftlichen und rechtlichen) Denkansätze, die Geschlecht als anti-essentialistisch verstehen und damit verschiedene gesellschaftliche Positionierungen von Frauen und Männern nicht länger als Ergebnis biologischer Differenzen anerkennen (Lang und Peters 2018, S. 13). In diesem Kontext wird Antifeminismus als Kritik an den Gender-Studies untersucht, deren Infragestellung der Naturhaftigkeit der Zweigeschlechtlichkeit in den letzten Jahren heftige anti-genderistische Reflexe hervorgerufen hat (Hark und Villa 2015b; Wimbauer et al. 2015).

Diese Befunde zeigen, dass auch aktuelle antifeministische Positionen in breitere gesellschaftliche Diskurse eingebunden sind. Insbesondere betrifft dies das vorgebliche Verschwinden traditioneller Geschlechterordnungen, d.h. von „klassischen“ Väter- und Mütterrollen. Die mit dieser Geschlechterordnung verbundenen Machtverhältnisse seien durch feministische Bewegungen nachhaltig infrage gestellt und würden als strukturell prekär erlebt. 
Während für die Wilhelminische Zeit die Einbindung des Antifeminismus in antimoderne Weltanschauungssysteme nachgewiesen wurde, gilt der moderne Antifeminismus zwar als hochgradig anschlussfähig an aktuelle konservative und rechtspopulistische Diskurse (Petchesky 1981; Keskinen 2013; Grigat 2017; Hentges und Nottbohm 2017; Schmincke 2019; Kaiser 2020), entsprechende empirisch-quantitative Analysen existieren jedoch bisher kaum. Insbesondere die Frage, inwiefern diese Diskurse der Verunsicherung und der Wunsch nach Restitution traditioneller Geschlechter- und damit verbundener Welt-Ordnungen auf etablierten Einstellungsclustern beruhen, ist derzeit noch wenig untersucht.

\section{Antifeminismus und seine Einbettung in weltanschauliche Perspektiven}

Die bisherigen Studien legen nahe, dass antifeministische Positionen in umfassendere Einstellungscluster eingebettet sind. Um diese Konstellation näher zu bestimmen, greifen wir auf ein an Karl Mannheim (2002; [1936], S. 2f.) angelehntes Konzept der Weltanschauung zurück. ${ }^{11}$ Soziologisch lassen sich Weltanschauungen als sozial geteilte Überzeugungssysteme auffassen (Beyer und Schnabel 2019). In Anlehnung an Mannheim nehmen wir an, dass Weltanschauungen Wahrnehmungen, Erleben, Bewertungen und Handlungsdispositionen mit sozial anschlussfähigem Sinn verbinden. Weltanschauungen sind Überzeugungssysteme, die nicht nur bestimmte Lebensbereiche, sondern alle Sphären des Lebens durchdringen. In Erweiterung von Mannheim verortet Schütz (Schütz und Luckmann 2003; [1973/84], S. 29ff.) Weltanschauungen in der sozial geteilten Alltagswelt - ,der Welt in Reichweite“. In dieser Alltagswelt begegnen wir den Mitmenschen, die unsere Ansichten teilen und mit uns in einem gemeinsamen kulturellen Rahmen interagieren (Schütz und Luckmann 2003; [1973/84], S. 35). Weltanschauungen sind relativ stabil. Wenn sich keine Unterbrechungen ereignen, prozessieren Akteur*innen auf der Basis von Routinen quasi-automatisch situative Informationen und hinterfragen sie nicht (Schütz und Luckmann 2003; [1973/84], S. 156ff.). Dadurch bestätigen und stützen Akteur*innen gleichzeitig ihre Weltanschauungen. ${ }^{12}$ Weltanschauungen, könnte man sagen, transzendieren Interpretationen von Situationen und bieten fundamentale Orientierungsschemata.

Im Folgenden wird empirisch untersucht, ob antifeministische Einstellungen auch in solche umfassenderen Orientierungsschemata eingebunden sind. Unsere Studie hat stark explorativen Charakter, sodass die empirischen Analysen nicht als strenger

\footnotetext{
11 Eine ausführliche Darstellung der Geschichte des Begriffs und seiner Abgrenzung zum Begriff der Ideologie findet sich u. a. bei Berger und Luckmann (1967, S. 1-20) oder bei Schützeichel (2012). Der Weltanschauungsbegriff weist zwar deutliche Überschneidungen mit Mannheims ,totalem“ Ideologiebegriff auf - im Sinne einer ,totalen Bewußtseinsstruktur [eines] Zeitalters bzw. [einer historisch-sozial konkret bestimmten] Gruppe“ (Mannheim 1929, S. 8). Aber ob es sich hier um „objektiv notwendiges und zugleich falsches Bewusstsein“ (Adorno und Horkheimer 1956, S. 168) im Sinne einer an Marx orientierten Ideologiekritik handelt, ist eine offene Frage. Deshalb verwenden wir hier den weniger voraussetzungsvollen Begriff der Weltanschauung.

12 Manchmal entstehen jedoch Inkonsistenzen zwischen Realität und Erwartung - in sozialen Settings sind Akteur*innen dann daran interessiert, diese Inkonsistenzen zu ,normalisieren“ (Garfinkel 1967).
} 
Hypothesentest zu verstehen sind. Wir knüpfen aber an bisherige Studienergebnisse an, die zum einen die Nähe des Antifeminismus zu rechtspopulistischen Positionen mit qualitativen Untersuchungen nachweisen (s. oben) und zum anderen in Bezug auf den verwandten (aber nicht identischen) Komplex des Sexismus die Nähe zu Dominanzorientierungen, autoritären Orientierungsmustern und Anomiewahrnehmungen mit standardisierten Daten ermittelt haben (Endrikat 2003).

Wir destillieren aus dieser Forschung fünf Bestandteile einer konservativ-traditionalistischen Weltanschauung: Wahrnehmungen und Überzeugungen bezüglich (i) der Geschwindigkeit des sozialen Wandels und der individuellen Kontrolle seiner Folgen (Anomia), (ii) dem Stellenwert politischer Führungspersönlichkeiten (Autoritarismus), (iii) der eigenen Kraft, Herausforderungen erfolgreich bewältigen zu können (Selbstwirksamkeit), (iv) der Überlegenheit bestimmter sozialer Gruppen (Soziale Dominanzorientierung) sowie (v) der Vertrauenswürdigkeit fremder Menschen (Vertrauen in Fremde). Diese fünf Weltanschauungsbestandteile durchziehen alle Sphären des Lebens; sie sind relevant dafür, wie Menschen den Anforderungen spätmoderner Gesellschaften begegnen und wie Alltagsphänomene und Interaktionen mit Mitmenschen sinnhaft gedeutet werden.

I. Auf die Relevanz, die der Wahrnehmung der Geschwindigkeit und Kontrollierbarkeit sozialen Wandels zukommt, wies Srole (1956) bereits 1956 hin. Die von ihm entwickelte Anomia-Skala gilt inzwischen als reliables Messinstrument individuellen Anomie-Empfindens und als Erklärungsfaktor für Vorurteile und gruppenbezogene Menschenfeindlichkeit (Hüpping und Reinecke 2007; Heyder und Gaßner 2012). Wir verwenden diese Skala, um zu testen, ob sich antifeministische Einstellungen gegen die Veränderung hierarchisch-binärer Geschlechterverhältnisse richten und mit der negativen Bewertung eines progressiven Gesellschaftswandels korreliert sind.

II. Ähnlich sind individuelle Positionierungen zu Normbefolgung und autoritärer Führung wichtige Weltanschauungsbestanteile (Adorno et al. 1950). Auch in diesem Fall existiert eine über Jahrzehnte hinweg weiterentwickelte Skala, die Autoritarismus als individuelles Einstellungscluster erfassen soll (Beierlein et al. 2014). Autoritäre Einstellungen gelten als starke Prädiktoren für Vorurteile und Diskriminierung. Wir verwenden diese Skala, um zu ergründen, ob antifeministische Einstellungen demokratischen Gesellschaftsstrukturen und der damit verbundenen (institutionellen) Anerkennung von Differenzen entgegenstehen.

III. Mit den beiden ersten Weltanschauungsbestandteilen hängt die Wahrnehmung von Selbstwirksamkeit eng zusammen. Rotter entwickelte (1966) eine Skala zur Messung des locus of control. Der locus of control bezeichnet die Überzeugung, dass das eigene Handeln die Zielerreichung beeinflussen kann. Diese Überzeugung gilt als Determinante für Erfolgserwartungen und damit für die Ausführungsbereitschaft für zielverwirklichende Handlungen (u.a. Lange und Tiggemann 1981; Reich und Infurna 2017). Wir verwenden die entsprechende Messskala und vermuten, dass antifeministische Einstellungen mit externalen Kontrollüberzeugungen verbunden sind, also mit Überzeugungen, dass Ereignisse der eigenen Kontrolle entzogen sind. So scheint etwa in der neuen Männerbewe- 
gung die Überzeugung zu dominieren, gegen die u. a. durch die Rechtsprechung gestützte Durchsetzung von „feministischen Interessen“ machtlos zu sein.

IV. Eine ausgeprägte soziale Dominanzorientierung fordert, dass die Gesellschaft hierarchisch gegliedert sein soll. Zur Erfassung der Sozialen Dominanzorientierung wurde eine Skala entwickelt, um Einstellungen gegenüber Intergruppenbeziehungen zu erfassen (Pratto et al. 1994). Wir nutzen diese Skala, um zu ermitteln, inwiefern eine solche gruppenbezogene Dominanzorientierung mit antifeministischen Einstellungen zusammenhängt. Wir vermuten, dass die mit antifeministischen Einstellungen verbundene Biologisierung sozialer Positionierung Antifeminismus kognitiv mit sozialer Dominanz und Rassismus verknüpft.

V. Sozialwissenschaftlich gesehen ist Vertrauen eine wichtige soziale Ressource, die Kooperation ermöglicht (Hardin 2002). Vertrauen kann in konkrete Andere oder generalisierte Andere in Form von Institutionen gesetzt werden. Die Messung des Vertrauens in Fremde ist ein valider Prädiktor für soziale Kooperationsbereitschaft und für die Akzeptanz gesellschaftlicher Institutionen. Wir verwenden hier eine ebenfalls bereits getestete Skala (Jagodzinski und Manabe 2004) und vermuten, dass fehlendes Vertrauen in Fremde auch mit Vorurteilen gegen Menschen mit anderen - z. B. feministischen - Überzeugungen korreliert ist. Den gemeinsamen Nenner stellt hier eine wahrgenommene und grundsätzlich abgelehnte Differenz zum Selbst dar.

Der folgende, empirische Teil des Artikels analysiert dementsprechend antifeministische Einstellungen in Deutschland und kontextualisiert diese im Rahmen solchermaßen spezifizierter Weltanschauungen.

\section{Antifeminismus in Deutschland: Daten und Ergebnisse}

\subsection{Antifeministische Einstellungen}

Die hier präsentierten Auswertungen liefern einen Überblick über die Verbreitung und weltanschauliche Einbettung antifeministischer Einstellungen. Einstellungen haben sowohl kognitive als auch affektive und konative Dimensionen, die potenziell handlungsrelevant werden können; daraus ergibt sich ihre soziologische und soziale Relevanz.

Die Stichprobe umfasst 1202 Befragte, die zwischen August und September 2016 in Form einer schriftlichen, standardisierten Online-Befragung interviewt wurden. Dafür haben wir mit einem professionellen Online-Panel-Anbieter zusammengearbeitet. Die Mitglieder des Panels werden sowohl online als auch offline aktiv rekrutiert. Die Qualität der Daten wird vonseiten des Anbieters basierend auf der ISO Norm 26362 garantiert. Die Befragten unseres Datensatzes wurden zufällig aus dem Mitglieder-Pool des Panel-Anbieters ausgewählt. Um die deutsche Gesamtbevölkerung einigermaßen verlässlich abzubilden, wurden Quoten hinsichtlich Geschlecht (49,8\% weiblich), Alter $(m=42,31 ; s=13,73)$, Bildung $(1,5 \%$ kein Abschluss; 31,4\% Hauptschulabschluss; 34,9\% Realschulabschluss; 9,9\% Fachhochschulreife; 22,8\% allgemeine Hochschulreife) und Region (17,3\% Ostdeutsche) 
festgelegt. Aufgrund der Selektivität von Online-Samples kann jedoch nicht von einer ,repräsentativen“ Stichprobe der deutschen Bevölkerung im strengen Sinne gesprochen werden.

Antifeminismus wurde über sieben Einzel-Items erfasst, die sich auf Feminismus als gesellschaftsverändernde Kraft beziehen. ${ }^{13}$ Diese Items konnten auf einer Antwortskala von „1“ (stimme überhaupt nicht zu) bis ,6“ (stimme voll zu) bewertet werden. Der Index selbst ist eine übersetzte und gekürzte Version eines bereits getesteten Befragungsinstruments (Fassinger 1994). Das Ziel des Messinstruments von Fassinger ist eine reliable und valide Messung der Einstellungen zum Feminismus. Eine negative Einstellung misst antifeministische Einstellungen in unserem Sinne, wobei die Stärke der Negativität sowohl durch die Abstufung der Einzelitems als auch die Abstufung der Bewertungsskala erreicht wird. Abb. 1 zeigt die Einzelitems und die jeweiligen Zustimmungswerte. Dafür wurden die drei Zustimmungs- bzw. Ablehnungskategorien zusammengefasst. Das Nichtvorliegen antifeministischer Einstellungen bedeutet jedoch nicht, dass die Befragten eine profeministische Haltung hätten; lediglich, dass antifeministische Einstellungen abgelehnt werden.

Abb. 1 zeigt, dass den drei sich positiv auf den Feminismus beziehenden Items ca. 51,0 bis 55,9\% der Befragten (voll bis überwiegend) zugestimmt haben. Für die vier negativ formulierten Aussagen sind die Unterschiede der Zustimmungswerte deutlich größer: Der Aussage ,es gibt bessere Möglichkeiten für Frauen für Gleichberechtigung zu kämpfen als den Feminismus“" stimmen 57,6\% zu, während lediglich 19,4\% finden, , der Feminismus [sei] eine Bedrohung für die gesellschaftliche Ordnung“.

Für die weiteren Analysen wurden die ursprünglichen intervallskalierten Werte zu einem additiven Gesamtindex zusammengefasst, ${ }^{14}$ der auf einen Wertebereich zwischen 0 und 1 standardisiert ist $(, 0 “=$ nicht antifeministisch; , $1 "=$ sehr stark antifeministisch). Es ist diese Index-Variable, die letztlich antifeministische Einstellungen abbilden soll, nicht die jeweils isolierten Items. Die Variable ist normalverteilt $(\mathrm{m}=0,58 ; \mathrm{s}=0,14 ; n=1174)$. Knapp über $9 \%$ der Befragten liegen im oberen Quartil, sind also vergleichsweise stark antifeministisch eingestellt.

\subsubsection{Soziodemografische Verteilung antifeministischer Einstellungen}

Wer sind die Personengruppen, die eine antifeministische Einstellung aufweisen? Hierfür werfen wir einen Blick auf relevante soziodemografische Merkmale: Tab. 1

\footnotetext{
13 Dabei wurde bewusst offengelassen, was genau unter „Feminismus“ zu verstehen ist, um den Assoziationen der Befragten Raum zu lassen. Es geht also um die Vermessung von Assoziationen zum Feminismus, die die Befragten selbst haben, nicht um die Abfrage einer ,richtigen“ oder „wahren“ Auffassung (einer bestimmten Form) von Feminismus. Die isolierten Einzelitems messen dabei nur einzelne Aspekte des Antifeminismus und dies in unterschiedlicher Intensität - von leichter Kritik des Feminismus als Strategie, um „für Gleichberechtigung zu kämpfen“ bis hin zur wahrgenommenen „Bedrohung für die gesellschaftliche Ordnung“. Erst der Gesamtindex ergibt ein reliables und valides Messinstrument.

14 Die Ergebnisse der Hauptkomponentenanalyse können hier aus Platzgründen nicht berichtet werden, die Autor*innen stellen diese auf Anfrage zur Verfügung. Die Reliabilitätsanalyse ergab einen CronbachsAlpha-Wert von 0,8 .
} 
astimme nicht zu $\quad$ stimme zu

Es gibt bessere Möglichkeiten für Frauen für Gleichberechtigung zu kämpfen als den Feminismus.

Mehr Menschen würden den Feminismus befürworten, wenn sie mehr darüber wüssten.

Feministinnen sind in ihren Ansichten zu radikal und zu extrem.

Feministische Bewegungen haben schon viel Positives in Sachen Gleichberechtigung erreicht.

Feministische Perspektiven sollten in allen gesellschaftlichen Bereichen berücksichtigt werden.

Feministinnen sind zu realitätsfremd für die wirkliche Welt

Der Feminismus ist eine Bedrohung für die gesellschaftliche Ordnung.

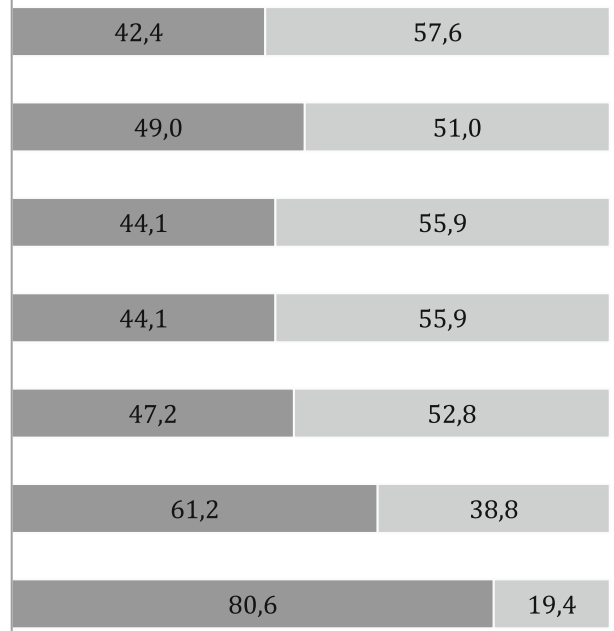

Abb. 1 Deskriptive Verteilung antifeministischer Einstellungen. (Anmerkungen: prozentuale Häufigkeiten; Ablehnungs- und Zustimmungskategorien zusammengefasst)

zeigt Gruppenmittelwerte hinsichtlich des berichteten Geschlechts, des Alters, Bildungsabschlusses, Wohnorts (Ost- oder Westdeutschland), Konfession sowie des Familienstands. Laut den Ergebnissen einer einfaktoriellen ANOVA sind die Gruppenunterschiede jedoch nur für die Geschlechtergruppen $\left(\mathrm{Eta}^{2}=0,077, p<0,000\right)$, den Wohnort $\left(\mathrm{Eta}^{2}=0,005 ; p<0,015\right)$ und den Familienstand $\left(\mathrm{Eta}^{2}=0,007 ; p<0,017\right)$ signifikant: Männer, Personen aus Ostdeutschland und Geschiedene äußern demnach häufiger eine antifeministische Einstellung als die jeweiligen Vergleichsgruppen.

Die Untersuchung der Parteipräferenz (hier nicht aufgeführt) birgt wenig Überraschendes: Die Mittelwerte für antifeministische Einstellungen liegen bei CDUund SPD-nahen Personen nah beieinander $(m=0,60$ und $m=0,58)$, sie sind für Personen, die die Partei der Grünen präferieren, besonders niedrig $(m=0,53)$ und für AfD- und NPD-Sympathisanten besonders hoch $(m=0,64$ und $m=0,65)$. Die Zwischengruppen-Differenzen der unterschiedlichen Parteien-Präferenzen sind signifikant $\left(\mathrm{Eta}^{2}=0,008 ; p<0,000\right)$.

\subsubsection{Antifeminismus als Element einer hierarchisch-binären Geschlechterordnung}

Wir argumentierten oben, dass Antifeminismus in Überzeugungen über die traditionelle, hierarchisch-binäre Geschlechterordnung eingebettet ist. Empirisch getestet wird dies, indem wir überprüfen, wie stark der Antifeminismus-Index mit Einstellungen zur Geschlechterordnung korreliert ist. Hierfür nutzen wir folgende Indices:

- Index, der erfasst, ob Gleichstellung bereits erreicht ist (Eckes und Six-Materna 1998),

- Index zur Einstellung zu Gleichstellungsgesetzen (Eckes und Six-Materna 1998), 
Tab. 1 Gruppenmittelwerte antifeministischer Einstellungen nach soziodemografischen Merkmalen

\begin{tabular}{|c|c|c|c|c|c|c|}
\hline & Merkmal & Mittelwert & $\begin{array}{l}\text { Standard- } \\
\text { abweichung }\end{array}$ & $N$ & $\begin{array}{l}\text { Eta-Quadrat- } \\
\text { Koeffizient }\end{array}$ & $p$ \\
\hline \multirow[t]{2}{*}{ Geschlecht } & Weiblich & 0,54 & 0,13 & 579 & \multirow[t]{2}{*}{0,077} & \multirow[t]{2}{*}{0,001} \\
\hline & Männlich & 0,62 & 0,14 & 595 & & \\
\hline \multirow[t]{5}{*}{ Altersgruppe } & $17-30$ & 0,59 & 0,13 & 264 & \multirow[t]{5}{*}{0,002} & \multirow[t]{5}{*}{0,705} \\
\hline & $31-40$ & 0,58 & 0,13 & 215 & & \\
\hline & $41-50$ & 0,58 & 0,14 & 260 & & \\
\hline & $51-60$ & 0,58 & 0,15 & 273 & & \\
\hline & $61-70$ & 0,57 & 0,14 & 162 & & \\
\hline \multirow{5}{*}{$\begin{array}{l}\text { Höchster } \\
\text { Schulab- } \\
\text { schluss }\end{array}$} & Ohne Abschluss & 0,62 & 0,20 & 18 & \multirow[t]{5}{*}{0,007} & \multirow[t]{5}{*}{0,084} \\
\hline & $\begin{array}{l}\text { Volks-/ } \\
\text { Hauptschule }\end{array}$ & 0,58 & 0,12 & 366 & & \\
\hline & $\begin{array}{l}\text { Mittlere Reife/ } \\
\text { Realschule }\end{array}$ & 0,58 & 0,13 & 402 & & \\
\hline & Fachhochschulreife & 0,60 & 0,15 & 116 & & \\
\hline & Abitur & 0,56 & 0,17 & 269 & & \\
\hline \multirow[t]{2}{*}{ Region } & Westdeutschland & 0,58 & 0,14 & 970 & \multirow[t]{2}{*}{0,005} & \multirow[t]{2}{*}{0,015} \\
\hline & Ostdeutschland & 0,60 & 0,14 & 204 & & \\
\hline \multirow[t]{4}{*}{ Konfession } & Katholisch & 0,58 & 0,13 & 291 & \multirow[t]{4}{*}{0,002} & \multirow[t]{4}{*}{0,527} \\
\hline & Protestantisch & 0,57 & 0,13 & 311 & & \\
\hline & Andere & 0,59 & 0,18 & 61 & & \\
\hline & Keine Konfession & 0,58 & 0,15 & 510 & & \\
\hline \multicolumn{2}{|c|}{ Familienstand Ledig } & 0,58 & 0,15 & 414 & \multirow[t]{4}{*}{0,07} & \multirow[t]{4}{*}{0,017} \\
\hline & Verheiratet & 0,57 & 0,13 & 580 & & \\
\hline & Geschieden & 0,61 & 0,15 & 157 & & \\
\hline & Insgesamt & 0,58 & 0,14 & 1173 & & \\
\hline
\end{tabular}

- Index zur individuellen Akzeptanz von gleichgeschlechtlichen Partnerschaften (Homophobie) (Heyder et al. 2005),

- Index zur allgemeinen Einstellung gegenüber Frauen (Misogynie) (Eckes und SixMaterna 1999),

- Index zur Überzeugung, dass es allein zwei Geschlechter gibt (Nagoshi et al. 2008),

- Index zur Akzeptanz der traditionellen geschlechtsspezifischen Arbeitsteilung in der Familie (Braun 2014). ${ }^{15}$

Tab. 2 zeigt eine Übersicht über die Zusammenhänge (Pearson's r): Antifeminismus korreliert hoch mit einem stereotypen Frauenbild, demzufolge Frauen überempfindlich und schutzbedürftig, aber auch verführerisch und undankbar seien. Hierzu gehört die Überzeugung, dass in Deutschland die Gleichstellung von Männern und Frauen inzwischen erreicht und weitere Gesetzesinitiativen unnötig seien. Antifeministische Orientierungen hängen zudem mittelstark mit einer Präferenz für eine traditionelle geschlechtsspezifische Arbeitsteilung im Haushalt zusammen sowie mit

15 Die Einzel-Indikatoren zu den standardisierten Indices finden sich in Tab. 4. 
Tab. 2 Korrelationen zwischen Antifeminismus und Elementen binär-hierarchischer Geschlechterordnungen

\begin{tabular}{ll}
\hline Index & \\
\hline Gleichstellung ist bereits erreicht & $0,498 * *(1165)$ \\
Zweigeschlechtlichkeit & $0,378 * * *(1174)$ \\
Misogynie & $0,512 * * *(1157)$ \\
Homophobie & $0,324 * * *(1148)$ \\
Geschlechtsspezifische Arbeitsteilung in der Familie & $0,292 * * *(1171)$ \\
Notwendigkeit gesetzlicher Regelungen & $-0,503 * * *(1169)$ \\
\hline
\end{tabular}

Pearson's r, Fallzahl in Klammern

$* * * p<0,001 ; * * p<0,01 ; * p<0,05$

der Ablehnung von Homosexualität und der Zurückweisung der Position, es gäbe mehr als zwei (eindeutig disjunkte) Geschlechter.

Bereits Chafetz und Dworkin (1987, S. 43) hatten festgestellt, dass sich antifeministische Einstellungen an der Ideologie der traditionellen Kleinfamilie mit der ihr typischen Aufteilung von männlicher - öffentlich sichtbarer - Erwerbsarbeit und weiblicher - im Intimbereich des Privaten geleisteter - Reproduktionsarbeit orientieren. Das wird auch von anderen Studien zum Antifeminismus belegt (Lang und Peters 2018, S. 14ff.). Auch die in unserer Erhebung ermittelten Personen mit einer antifeministischen Einstellung sympathisieren mit einer traditionellen Geschlechterordnung und der damit verbundenen Heteronormativität. ${ }^{16}$ In diesem Zusammenhang muss die Frage nach den „Verlierern“ von sozialem Wandel gestellt werden: Auch wenn in Deutschland nicht primär die im ökonomischen Verteilungskampf Benachteiligten antifeministische Einstellungen zu äußern scheinen, so lassen sich doch in den hier berichteten Antwortmustern „Verlierer“ identifizieren: Es ist dies die Gruppe derer, die die traditionelle Geschlechterordnung als legitim erachtet und ihrer Selbstwahrnehmung nach durch deren Erosion (weniger monetäre als kognitive) Sicherheit, Ansehen und Privilegien zu verlieren scheint.

\subsubsection{Antifeministische Einstellungen und ihre Einbettung}

Um die Einbettung antifeministischer Einstellungen in eine umfassende konservativtraditionalistische Weltanschauung zu prüfen, haben wir in einem zweiten Schritt multiple lineare Regressionsmodelle geschätzt, die die internen Zusammenhänge zwischen den Weltanschauungskomponenten konstant halten. Hohe Korrelationen zeigen einen hohen Grad der Einbettung an, keine Korrelationen würden darauf hindeuten, dass Antifeminismus eine eigenständige kognitive Struktur und damit Weltanschauung darstellen würde.

Modell 1 in Tab. 3 stellt das Basis-Modell dar, das lediglich die soziodemografischen Kontrollvariablen umfasst, die sich in der bi-variaten Analyse als signifikant herausgestellt haben. In Modell 2 fügen wir zwei Konstrukte hinzu, die das Vertrauen in politische Institutionen und die Presse messen. Chafetz und Dworkin (1987) ver-

\footnotetext{
16 Mit Butler (1991) lässt sich Heteronormativität als gesellschaftlich hochgradig normiertes und abgesi-
} chertes Identifizieren von Zweigeschlechtlichkeit und Heterosexualität beschreiben. 
muteten, dass antifeministische Bewegungen eher ,pro-institutionell“ sind, da sie sich gegen anti-institutionell ausgerichtete feministische Anliegen richteten, während die Forschung zum aktuellen Antifeminismus nahelegt, dass sich Antifeminismus gegen staatliche Gleichstellungspolitiken richte, da diese vor allem Männer zu Verlierern mache. Diese Annahmen wollen wir im Folgenden testen. Weiterhin interessiert uns, inwiefern Weltanschauungsbestandteile mit antifeministischer Einstellung gekoppelt sind (Tab. 3, Modell 3). ${ }^{17}$

Tab. 3 zeigt, dass die gemessene Stärke einer antifeministischen Einstellung für Männer um 7,8 Prozentpunkte höher ist als für Frauen. Während Alter einen schwach signifikanten Einfluss hat, zeigen Einkommen und Bildung keine signifikanten Einflüsse. Personen aus Ostdeutschland sind antifeministischer als Personen aus Westdeutschland, und Geschiedene eher als Ledige. Schwach signifikant sind auch die Effekte der Variablen „Mitgliedschaften in Organisationen“ und ,,politische Selbsteinschätzung“. Die Eingebundenheit in Organisationen, seien es politische oder Freizeit-Organisationen, zeigen den aus der Sozialkapital-Theorie seit Langem bekannten Effekt (Putnam 2000; Pickel und Gladkich 2012): Soziale Einbindung erhöht die Akzeptanz für die Anliegen anderer; in diesem Fall: feministischer Anliegen. Auch der Effekt der politischen Selbsteinschätzung stimmt mit gängigen Annahmen überein: Personen, die sich eher politisch ,,rechts“ einordnen, äußern sich stärker antifeministisch. Dies unterstützt die oben berichteten Befunde zur Parteien-Präferenz.

In Modell 2 werden die Variablen „Vertrauen in die Presse“ und in ,,politische Institutionen“ (Regierung, Parlament und Parteien) in den Blick genommen. Beide Variablen weisen negative Effekte auf: Je geringer das Vertrauen in Presse und in politische Institutionen, desto stärker werden antifeministische Einstellungen geäußert. Der Befund widerspricht auf den ersten Blick den Analysen von Chafetz und Dworkin (1987, S. 35) und bestätigt die Untersuchungen zum aktuellen Antifeminismus: Antifeminismus ist in Deutschland nicht pro-institutionell. Dies deutet darauf hin, dass Menschen mit antifeministischen Ansichten vermuten, dass feministische Belange ,die Institutionen dominieren“. Auch die Anliegen der starken autonomen Frauenbewegung der 1970er- und 1980er-Jahre, die zunächst explizit gegen die bestehenden Institutionen lanciert wurden, scheinen nun im öffentlichen und politischen Mainstream angekommen zu sein.

Dieser teils imaginierte, teils reale institutionelle Wandel korrespondiert mit der Wahrnehmung der gesamtgesellschaftlichen Verhältnisse. Modell 3 zeigt, dass die eingeführten Indices ausgesprochen erklärungskräftig sind und dazu führen, dass nicht nur die Unterschiede zwischen Ost- und Westdeutschland insignifikant werden, sondern dass auch die Rolle der Mitgliedschaften in Organisationen sowie das Misstrauen in Presse und politische Institutionen durch diese Variablen mediiert werden. ${ }^{18}$ Erwartungsgemäß hängen hohes Anomie-Empfinden, autoritäre Überzeu-

\footnotetext{
17 Eine Auflistung der Skalen und ihrer Items findet sich in Tab. 5 des Anhangs.

18 Die Variablen Anomie, Soziale Dominanzorientierung und Selbstwirksamkeit sind signifikant negativ, die Variable Vertrauen in Fremde signifikant positiv mit dem Vertrauen in Presse und in politische Institutionen korreliert (hier nicht berichtet, Ergebnisse auf Anfrage). Allein der Effekt des AutoritarismusIndexes ist nicht signifikant.
} 
Tab. 3 OLS-Regressionsmodelle zur Erklärung antifeministischer Einstellungen

\begin{tabular}{|c|c|c|c|}
\hline & Modell 1 & Modell 2 & Modell 3 \\
\hline \multirow[t]{2}{*}{ (Konstante) } & $0,376^{* * *}$ & $0,434 * * *$ & $0,352 * * *$ \\
\hline & $(0,028)$ & $(0,03)$ & $(0,046)$ \\
\hline \multirow[t]{2}{*}{ Geschlecht (Ref. Frauen) } & $0,078 * * *$ & $0,081 * * *$ & $0,081 * * *$ \\
\hline & $(0,008)$ & $(0,008)$ & $(0,008)$ \\
\hline \multirow[t]{2}{*}{ Alter } & $-0,009 *$ & $-0,008^{*}$ & $-0,008^{*}$ \\
\hline & $(0,004)$ & $(0,004)$ & $(0,003)$ \\
\hline \multirow[t]{2}{*}{ Bildung } & $-0,003$ & 0,001 & 0,007 \\
\hline & $(0,004)$ & $(0,004)$ & $(0,004)$ \\
\hline \multirow[t]{2}{*}{ Einkommen } & 0,001 & 0,001 & 0,001 \\
\hline & $(0,001)$ & $(0,001)$ & $(0,001)$ \\
\hline \multirow[t]{2}{*}{ Region (Ref. Westdeutschland) } & $0,032 * *$ & $0,025^{*}$ & 0,018 \\
\hline & $(0,011)$ & $(0,011)$ & $(0,011)$ \\
\hline \multirow[t]{2}{*}{ Familienstand: verheiratet (Ref. „ledig“) } & $-0,004$ & $-0,006$ & $-0,01$ \\
\hline & $(0,011)$ & $(0,01)$ & $(0,01)$ \\
\hline \multirow[t]{2}{*}{ Familienstand: geschieden (Ref. „ledig“) } & $0,036^{* *}$ & $0,033 * *$ & $0,036 * *$ \\
\hline & $(0,015)$ & $(0,014)$ & $(0,014)$ \\
\hline \multirow{2}{*}{$\begin{array}{l}\text { Mitgliedschaft in Organisationen } \\
\text { (Anzahl) }\end{array}$} & $-0,007 *$ & $-0,006$ & 0,001 \\
\hline & $(0,004)$ & $(0,004)$ & $(0,004)$ \\
\hline \multirow[t]{2}{*}{ Links-Rechts-Einschätzung } & $0,016^{* * * *}$ & $0,014 * * *$ & $0,007 *$ \\
\hline & $(0,002)$ & $(0,002)$ & $(0,002)$ \\
\hline \multirow[t]{2}{*}{ Vertrauen in die Presse } & - & $-0,014 *$ & $-0,010$ \\
\hline & & $(0,007)$ & $(0,006)$ \\
\hline \multirow[t]{2}{*}{ Vertrauen in politische Institutionen } & - & $-0,006^{*}$ & 0,001 \\
\hline & & $(0,002)$ & $(0,003)$ \\
\hline \multirow[t]{2}{*}{ Anomie } & - & - & $0,070 *$ \\
\hline & & & $(0,029)$ \\
\hline \multirow[t]{2}{*}{ Autoritarismus } & - & - & $0,081 * *$ \\
\hline & & & $(0,03)$ \\
\hline \multirow[t]{2}{*}{ Selbstwirksamkeit } & - & - & $-0,066 * * *$ \\
\hline & & & $(0,019)$ \\
\hline \multirow[t]{2}{*}{ Soziale Dominanzorientierung } & - & - & $0,124 * * *$ \\
\hline & & & $(0,026)$ \\
\hline \multirow[t]{2}{*}{ Vertrauen in Fremde } & - & - & $-0,041 *$ \\
\hline & & & $(0,019)$ \\
\hline$n$ & 1022 & 1022 & 1022 \\
\hline $\mathrm{R}^{2}$ & 0,146 & 0,165 & 0,236 \\
\hline
\end{tabular}

Nicht-standardisierte Koeffizienten, Standardfehler in Klammern $* * * p<0,001, * * p<0,01, * p<0,05$

gungen und eine ausgeprägte soziale Dominanzorientierung eng mit einer antifeministischen Einstellung zusammen. Das gilt auch für mangelndes Vertrauen in Fremde und mangelnde empfundene Selbstwirksamkeit (bzw. hohe „externale Kontrollüberzeugungen“; Rotter 1966). Antifeminismus ist demnach stark mit jenen fünf 
Bestandteilen einer konservativ-traditionalistischen Weltanschauung verknüpft, die wir oben eingeführt haben.

Diese Befunde zeigen, dass aktuelle antifeministische Überzeugungen in Deutschland nicht nur in ein umfassendes Einstellungscluster eines hierarchisch-binären Geschlechtermodells eingebettet sind, sondern sich auch als Reaktion auf als negativ empfundene Folgen des institutionellen und gesamtgesellschaftlichen Wandels - wie Kontingenz der Lebensläufe, gesellschaftliche Beschleunigung, Demokratisierung, Gleichstellung benachteiligter Gruppen - verstehen lassen.

\section{Fazit und Diskussion}

Wir argumentieren in diesem Beitrag, dass Antifeminismus seit Beginn der Frauenbewegung ein Phänomen von gesellschaftlicher Tragweite war und ist, und aktuell insbesondere auf die institutionellen Erfolge der Frauenbewegung reagiert. Die Bewegungsforschung zu Gegenmobilisierung zeigt, dass Antifeminismus dann aktiviert wird, wenn sich feministische Belange erfolgreich in politischen Arenen etablieren können. Antifeministische Initiativen galten lange als pro-institutionell und schienen ihre Aktivist*innen und Sympathisant*innen vor allem unter denjenigen zu finden, die befürchten, ihre gesellschaftliche Position durch die Emanzipation von Frauen zu verlieren (z.B. durch die Erosion der traditionellen Familienstruktur).

Die empirische Analyse unserer Umfragedaten ergab einige Besonderheiten des Antifeminismus in Deutschland: Auch wenn unsere Ergebnisse, wie die der meisten Online-Befragungen, keine Repräsentativität beanspruchen können, deuten sie doch darauf hin, dass sich in Deutschland kaum soziodemografische Gruppen identifizieren lassen, für die die Äußerung antifeministischer Einstellungen typisch wären. Antifeministische Einstellungen finden sich vielmehr in fast allen gesellschaftlichen Schichten. Dies legt nahe, dass Antifeminismus keine eigenständige Weltanschauung im Sinne einer gruppenspezifischen Bewusstseinsstruktur darstellt, sondern vielmehr Bestandteil einer umfassenderen konservativ-traditionalistischen Weltanschauung ist. Dies zeigt sich zum einen an dem sich bereits in anderen Studien (Lang und Peters 2018) andeutenden Zusammenhang mit konservativen Einstellungen zur Geschlechterordnung. Wir finden in unseren Daten starke Korrelationen, die zeigen, dass Antifeminismus hochgradig assoziiert ist mit fundamentaler Kritik an (institutionalisierten) Gleichstellungspolitiken sowie der normativen Überzeugung von der Richtigkeit eines traditionellen, hierarchisch-binären, heterosexuell geprägten Geschlechterverhältnisses, das als „,natürlich“ und „,richtig“ ontologisiert wird. In diesem Sinne ist Antifeminismus fester Bestandteil eines umfassenderen (geschlechter-)konservativen Einstellungssyndroms.

Zum anderen fanden wir signifikante Zusammenhänge mit fünf Grundbestandteilen einer konservativ-traditionalistischen Weltanschauung. Antifeministische Einstellungen sind mit Empfindungen von Anomie und Ohnmacht, autoritären Positionen, mangelnder Selbstwirksamkeit, sozialer Dominanzorientierung und Misstrauen in Fremde verbunden.

Der solchermaßen konkretisierte Antifeminismus lässt sich damit als Ausdruck eines Abwehrmechanismus gegenüber ,,progressiv-liberalen“ Weltanschauungen be- 
greifen. Auch wenn sich nicht bestätigt, dass es sich bei den Akteur*innen mit antifeministischen Einstellungen um „Modernisierungsverlierer“ handelt, kristallisiert sich hier eine Gruppe von Personen heraus, die sich durch den Wandel gängiger Interpretations- und Bewertungsmuster benachteiligt fühlt. Insofern Veränderungen durch die rechtliche Gleichstellung von Männern und Frauen und durch die Inklusion von Frauen in alle gesellschaftlichen Teilbereiche Veränderungen gesellschaftlicher Interpretationen und Wertmaßstäbe nach sich ziehen, wird diesen Veränderungen mit Skepsis und Ablehnung begegnet, da als natürlich erscheinende Grundpfeiler der (geschlechtlichen) Gesellschaftsordnung scheinbar erschüttert werden und damit die soziale Unsicherheit (und die Unsicherheit über die eigene gesellschaftliche Position) steigt. Die politische Forderung nach Geschlechtergerechtigkeit ist damit wichtiges „Kampfgebiet“, wenn es um Deutungshoheiten und die Verteilung von Deutungsmacht geht.

Der aktuelle Antifeminismus ist zudem mit einem grundlegend anti-institutionellen Impuls verknüpft, der indirekt für den wahrgenommenen institutionellen Erflog der Frauenbewegung spricht: Antifeminismus speist sich, basierend auf der Unterstellung, der Feminismus habe die politischen Institutionen und die Presse „,unterwandert“, aus einem gegen die „politischen Eliten“ gerichteten Impuls. In diesem Zusammenhang bestehen erkennbare Anknüpfungspunkte zu rechts-populistischen und -radikalen Positionen (Krause et al. 2015; Hentges und Nottbohm 2017; Stögner 2017; Kaiser 2020).

Der deutsche Antifeminismus, wie er sich uns in den Befragungsdaten präsentiert, kann damit als weltanschaulich fundierte Reaktion auf die vorgeblich in „Unordnung" geratenen Geschlechter- und Gesellschaftsverhältnisse verstanden werden. Er ist anschlussfähig für neurechte, aber aufgrund seines traditionell hierarchischbinären Geschlechterbildes potenziell auch für religiös-fundamentalistische Bewegungen (Kinnvall 2004; Näser-Lather et al. 2019; für den internationalen Vergleich siehe Behrensen et al. 2019). Inwiefern die vorgestellten Ergebnisse sich für andere Länder-Kontexte bestätigen lassen, kann zu diesem Zeitpunkt nur vermutet werden. Der vorliegende Beitrag dürfte jedoch die Virulenz und Tragweite des Phänomens aufgezeigt haben und soll weitere Studien motivieren. 


\section{Anhang}

Tab. 4 Indices zur Akzeptanz der Geschlechterordnung und ihre Indikatoren

\begin{tabular}{|c|c|c|}
\hline Index & Indikatoren & $\mathrm{m}(\mathrm{s})$ \\
\hline \multirow{4}{*}{$\begin{array}{l}\text { Gleichstellung } \\
\text { ist erreicht } \\
(0-1)\end{array}$} & Diskriminierung von Frauen ist in Deutschland immer noch ein Problem & \multirow{4}{*}{$\begin{array}{l}0,47 \\
(0,16)\end{array}$} \\
\hline & $\begin{array}{l}\text { Frauen und Männer haben in der heutigen Gesellschaft die gleichen } \\
\text { Chancen, etwas zu erreichen }\end{array}$ & \\
\hline & $\begin{array}{l}\text { Die Forderungen von Frauen nach Gleichberechtigung sind leicht nach- } \\
\text { zuvollziehen }\end{array}$ & \\
\hline & $\begin{array}{l}\text { Wenn Frauen tatsächlich einmal schlechter bezahlt werden als Männer, } \\
\text { dann nur deshalb, weil sie einfachere Arbeit zu leisten haben }\end{array}$ & \\
\hline \multirow{2}{*}{$\begin{array}{l}\text { Zweigeschlecht- } \\
\text { lichkeit } \\
(0-1)\end{array}$} & $\begin{array}{l}\text { Die Zweiteilung der Geschlechter von Mann und Frau ist von der Natur } \\
\text { vorgegeben }\end{array}$ & \multirow[t]{2}{*}{$\begin{array}{l}0,56 \\
(0,26)\end{array}$} \\
\hline & $\begin{array}{l}\text { Mit einer Person stimmt etwas nicht, wenn sie sagt, sie sei weder ein } \\
\text { Mann noch eine Frau }\end{array}$ & \\
\hline \multirow{3}{*}{$\begin{array}{l}\text { Homophobie } \\
(0-1)\end{array}$} & Es ist ekelhaft, wenn sich Homosexuelle in der Öffentlichkeit küssen & \multirow{3}{*}{$\begin{array}{l}0,37 \\
(0,24)\end{array}$} \\
\hline & Homosexualität ist unmoralisch & \\
\hline & $\begin{array}{l}\text { Ehen zwischen zwei Frauen bzw. zwischen zwei Männern sollten erlaubt } \\
\text { sein }\end{array}$ & \\
\hline $\begin{array}{l}\text { Misogynie } \\
(0-1)\end{array}$ & $\begin{array}{l}\text { Egal wie erfolgreich ein Mann auch sein mag, ohne eine Frau, die ihn } \\
\text { liebt, fehlt ihm etwas ganz Wichtiges }\end{array}$ & $\begin{array}{l}0,59 \\
(0,15)\end{array}$ \\
\hline
\end{tabular}

Die meisten Frauen interpretieren harmlose Äußerungen oder Handlungen als frauenfeindlich

Frauen sollten von Männern umsorgt und beschützt werden

Die meisten Frauen sehen gar nicht, was Männer alles für sie tun

Viele Frauen haben Spaß daran, mit Männern zu ,spielen“, indem sie sich zuerst verführerisch geben, dann aber die Annäherungsversuche der Männer zurückweisen

Geschlechts- $\quad$ Ein Kind, das noch nicht zur Schule geht, wird wahrscheinlich darunter spezifische Arbeitsteilung in der Familie $(0-1)$ leiden, wenn seine Mutter berufstätig ist

Alles in allem: Das Familienleben leidet darunter, wenn die Frau voll berufstätig ist

Einen Beruf zu haben ist ja ganz schön, aber das, was die meisten Frauen wirklich wollen, sind ein Heim und Kinder

Die Aufgabe des Mannes ist es, Geld zu verdienen, die der Frau, sich um Haushalt und Familie zu kümmern

Der Mann und die Frau sollten beide zum Haushaltseinkommen beitragen

Gleichstellungs- Alles in allem ist die Verabschiedung von Gleichstellungsgesetzen in Deutschland notwendig und sinnvoll

$(0-1)$

Frauen haben auf allen gesellschaftlichen Ebenen schon so viel erreicht, dass Sonderprogramme zu ihrer Förderung im Berufsleben überflüssig sind

Frauen und Männer haben in der Gesellschaft ganz unterschiedliche Aufgaben, sodass Gesetze zur Gleichstellung von Frauen und Männern einfach unsinnig sind 
Tab. 5 Weltanschauungs-Indices und deren Indikatoren

\begin{tabular}{|c|c|c|}
\hline Index & Indikatoren & $\mathrm{m}(\mathrm{s})$ \\
\hline \multirow[t]{4}{*}{$\begin{array}{l}\text { Anomie } \\
(0-1)\end{array}$} & $\begin{array}{l}\text { Wenn man die Ereignisse der letzten Jahre betrachtet, wird man rich- } \\
\text { tig unsicher }\end{array}$ & $\begin{array}{l}0,66 \\
(0,17)\end{array}$ \\
\hline & $\begin{array}{l}\text { Heute ändert sich alles so schnell, dass man oft nicht weiß, woran man } \\
\text { sich halten soll }\end{array}$ & \\
\hline & Moralische Grundsätze gelten heute nicht mehr & \\
\hline & Das Leben ist auch in der heutigen Zeit klar und geordnet & \\
\hline \multirow[t]{6}{*}{$\begin{array}{l}\text { Autoritarismus } \\
(0-1)\end{array}$} & $\begin{array}{l}\text { Gegen Außenseiter und Nichtstuer sollte in der Gesellschaft mit aller } \\
\text { Härte vorgegangen werden }\end{array}$ & $\begin{array}{l}0,57 \\
(0,16)\end{array}$ \\
\hline & $\begin{array}{l}\text { Unruhestifter sollten deutlich zu spüren bekommen, dass sie in der } \\
\text { Gesellschaft unerwünscht sind }\end{array}$ & \\
\hline & $\begin{array}{l}\text { Menschen sollten wichtige Entscheidungen in der Gesellschaft Füh- } \\
\text { rungspersonen überlassen }\end{array}$ & \\
\hline & $\begin{array}{l}\text { Wir sollten dankbar sein für führende Köpfe, die uns genau sagen, } \\
\text { was wir tun können }\end{array}$ & \\
\hline & Traditionen sollten unbedingt gepflegt und aufrechterhalten werden & \\
\hline & Bewährte Verhaltensweisen sollten nicht infrage gestellt werden & \\
\hline \multirow[t]{2}{*}{$\begin{array}{l}\text { Selbstwirksamkeit } \\
(0-1)\end{array}$} & $\begin{array}{l}\text { Leute wie ich haben sowieso keinen Einfluss darauf, was die Regie- } \\
\text { rung tut }\end{array}$ & $\begin{array}{l}0,42 \\
(0,25)\end{array}$ \\
\hline & Ich halte es für sinnlos, mich politisch zu engagieren & \\
\hline \multirow{4}{*}{$\begin{array}{l}\text { Soziale } \\
\text { Dominanz- } \\
\text { orientierung } \\
(0-1)\end{array}$} & $\begin{array}{l}\text { Wir sollten tun, was wir können, um gleiche Lebensbedingungen für } \\
\text { alle zu schaffen }\end{array}$ & $\begin{array}{l}0,76 \\
(0,17)\end{array}$ \\
\hline & Die Gleichwertigkeit aller Gruppen ist ein wichtiges Ideal & \\
\hline & Einige Gruppen sind einfach weniger wert als andere & \\
\hline & Unterlegene Gruppen sollten dort bleiben, wo sie hingehören & \\
\hline \multirow{2}{*}{$\begin{array}{l}\text { Vertrauen in } \\
\text { Fremde } \\
(0-1)\end{array}$} & Heutzutage kann man sich auf niemanden mehr verlassen & \multirow{2}{*}{$\begin{array}{l}0,28 \\
(0,23)\end{array}$} \\
\hline & $\begin{array}{l}\text { Wenn man mit Fremden zu tun hat, ist es besser, vorsichtig zu sein, } \\
\text { bevor man ihnen vertraut }\end{array}$ & \\
\hline
\end{tabular}

Funding Open Access funding enabled and organized by Projekt DEAL.

Open Access Dieser Artikel wird unter der Creative Commons Namensnennung 4.0 International Lizenz veröffentlicht, welche die Nutzung, Vervielfältigung, Bearbeitung, Verbreitung und Wiedergabe in jeglichem Medium und Format erlaubt, sofern Sie den/die ursprünglichen Autor(en) und die Quelle ordnungsgemäß nennen, einen Link zur Creative Commons Lizenz beifügen und angeben, ob Änderungen vorgenommen wurden.

Die in diesem Artikel enthaltenen Bilder und sonstiges Drittmaterial unterliegen ebenfalls der genannten Creative Commons Lizenz, sofern sich aus der Abbildungslegende nichts anderes ergibt. Sofern das betreffende Material nicht unter der genannten Creative Commons Lizenz steht und die betreffende Handlung nicht nach gesetzlichen Vorschriften erlaubt ist, ist für die oben aufgeführten Weiterverwendungen des Materials die Einwilligung des jeweiligen Rechteinhabers einzuholen.

Weitere Details zur Lizenz entnehmen Sie bitte der Lizenzinformation auf http://creativecommons.org/ licenses/by/4.0/deed.de. 


\section{Literatur}

Adorno, Theodor W., und Max Horkheimer. 1991. Ideologie. In Soziologische Exkurse, Hrsg. Institut für Sozialforschung, 162-181. Frankfurt am Main: Europäische Verlagsanstalt.

Adorno, Theodor W., Else Frenkel-Brunswik, Daniel J. Levinson, und Nevitt Sanford. 1950. The authoritarian personality. New York: Harper and Brothers.

Amlinger, Fabienne. 2015. Von weiblichem Schwachsinn, Blaustrümpfen und Frauen als Knalleffekt - die lange Tradition des Antifeminismus. Genderstudies 27:2-4.

Behrensen, Maren, Marianne Heimbach-Steins, und Linda E. Hennig (Hrsg.). 2019. Gender - Nation Religion: Ein internationaler Vergleich von Akteursstrategien und Diskursverflechtungen. Frankfurt am Main, New York: Campus.

Beierlein, Constanze, Frank Asbrock, Mathias Kauff, und Peter Schmidt. 2014. Die Kurzskala Autoritarismus (KSA-3): ein ökonomisches Messinstrument zur Erfassung dreier Subdimensionen autoritärer Einstellungen. GESIS-Working Papers 35. Mannheim: GESIS - Leibniz-Institut für Sozialwissenschaften.

Berger, Peter L., und Thomas Luckmann. 1967. Die gesellschaftliche Konstruktion der Wirklichkeit. Frankfurt am Main: Fischer.

Beyer, Heiko, und Annette Schnabel. 2019. The entanglement of religion and politics in Europe: how and why religious and political worldviews merge in times of uncertainty. Interdisciplinary Journal of Research on Religion 15(1):1-27.

Braukmann, Stephanie. 2003. „Die kapitalistische Gesellschaft schachert mit Allem und Jedem“. Die proletarisch-sozialistische Frauenbewegung im Kaiserreich und der Antisemitismus. Ariadne - Forum für Frauen- und Geschlechtergeschichte 43:6-13.

Braun, Michael. 2014. Gender-role attitudes (ISSP 94). Zusammenstellung sozialwissenschaftlicher Items und Skalen. ZIS - GESIS Leibniz Institute for the Social Sciences.

Butler, Judith. 1991. Das Unbehagen der Geschlechter. Frankfurt am Main: Suhrkamp.

Chafetz, Janet S., und Anthony G. Dworkin. 1987. In the face of threat: organised antifeminism in comparative perspective. Gender \& Society 1:33-60.

Dohm, Hedwig. 1902. Die Antifeministen: Ein Buch der Verteidigung. Berlin: Dümmler.

Drüeke, Ricarda. 2016. Digitale Öffentlichkeiten: Antifeminismus im Internet. http://www.gwi-boell.de/ sites/default/files/uploads/2016/07/digitale_offentlichkeiten_drueeke.pdf. Zugegriffen: 9. Aug. 2019.

Drüeke, Ricarda, und Elisabeth Klaus. 2014. Öffentlichkeiten im Internet: Zwischen Feminismus und Antifeminismus. FEMINA POLITICA - Zeitschrift für feministische Politikwissenschaft 23:59-70.

Drüeke, Ricarda, und Corinna Peil. 2015. Sprachliche Inklusion versus virtuellen Backlash. Über Antifeminismen im Internet. In Zwischen Gegebenem und Möglichem. Kritische Perspektiven auf Medien und Kommunikation, Hrsg. Ricarda Drüeke, Susanne Kirchhoff, Thomas Steinmaurer, und Martina Thiele, 275-288. Bielefeld: transcript.

Drüeke, Ricarda, und Elke Zobl. 2016. Online feminist protest against sexism: the German-language hashtag \#aufschrei. Feminist Media Studies 16:35-54.

Eckes, Thomas, und Iris Six-Materna. 1998. Leugnung von Diskriminierung. Eine Skala zur Erfassung des modernen Sexismus. Kölner Zeitschrift für Soziologie und Sozialpsychologie 29(3):224-238.

Eckes, Thomas, und Iris Six-Materna. 1999. Hostilität und Benevolenz. Eine Skala zur Erfassung des ambivalenten Sexismus. Zeitschrift für Sozialpsychologie 30(4):211-228.

Eisinger, Peter K. 1973. The conditions of protest behavior in American cities. The American Political Science Review 67:11-28.

Endrikat, Kirsten. 2003. Ganz normaler Sexismus. Reizende Einschnürung in ein Rollenkorsett. In Deutsche Zustände, Bd. 2, Hrsg. Wilhelm Heitmeyer, 120-141. Frankfurt am Main: Suhrkamp.

Fassinger, Ruth. 1994. Development and testing of the attitudes toward feminism and the women's movement (FWM) scale. Psychology of Women Quaterly 18(3):389-402.

Garfinkel, Harald. 1967. Studies in ethnomethodology. Cambridge: Polity Press.

Gerhard, Ute. 1996. Unerhört. Die Geschichte der deutschen Frauenbewegung. Reinbek bei Hamburg: Rowohlt.

Gesterkamp, Thomas. 2010. Geschlechterkampf von rechts. Wie Männerrechtler und Familienfundamentalisten sich gegen das Feindbild Feminismus radikalisieren. Bonn: Friedrich-Ebert-Stiftung.

Grigat, Stephan. 2017. AfD \& FPÖ. Antisemitismus, völkischer Nationalismus und Geschlechterbilder. Baden-Baden: Nomos.

Hardin, Russel. 2002. Trust and trustworthiness. New York: SAGE.

Hark, Sabine, und Paula-Irene Villa. 2015a. Anti-Genderismus. Bielefeld: transcript. 
Hark, Sabine, und Paula-Irene Villa. 2015b. „Eine Frage an und für unsere Zeit“. Verstörende Gender Studies und symptomatische Missverständnisse. In Anti-Genderismus, Hrsg. Sabine Hark, Paula-Irene Villa, 15-41. Bielefeld: transcript.

Hawley, George. 2017. Making sense of the alt-right. New York: Columbia University Press.

Hentges, Gudrun, und Kristina Nottbohm. 2017. Die Verbindung von Antifeminismus und Europakritik. Positionen der Parteien „Alternative für Deutschland“ und „Front National“. In Europäische Identität in der Krise?, Hrsg. Gudrun Hentges, Kristina Nottbohm, und Hans-Wolfgang Platzer, 167-208. Wiesbaden: Springer VS.

Herrmann, Steffen K. 2015. Politischer Antagonismus und sprachliche Gewalt. In Anti-Genderismus, Hrsg. Sabine Hark, Paula-Irene Villa, 79-92. Bielefeld: transcript.

Heyder, Aribert, und Anna Gaßner. 2012. Anomia, Deprivation und Werteorientierung zur Vorhersage rechtsextremistischer Einstellungen - Eine empirische Studie mit Repräsentativdaten aus Deutschland. Österreichische Zeitschrift für Politikwissenschaft 41(3):277-297.

Heyder, Aribert, Beate Küpper, und Andreas Zick. 2005. Dokumentation der Entwicklung und Überprüfung von Kurzskalen: Homophobie, Behinderten- und Obdachlosenabwertung. Bielefeld: GMF-Arbeitsbericht 2005/01.

Hüpping, Sandra, und Jost Reinecke. 2007. Abwärtsdriftende Regionen. Die Bedeutung sozioökonomischer Entwicklungen für Orientierungslosigkeit und Gruppenbezogene Menschenfeindlichkeit. In Deutsche Zustände, Bd. 5, Hrsg. Wilhelm Heitmeyer, 77-101. Frankfurt am Main: Suhrkamp.

Jagodzinski, Wolfgang, und Kazufumi Manabe. 2004. How to measure interpersonal trust? A comparison of two different measures. ZA-Information/Zentralarchiv für Empirische Sozialforschung 55:85-98.

Kaiser, Susanne. 2020. Politische Männlichkeit. Berlin: Suhrkamp.

Kemper, Andreas. 2011. [R]echte Kerle. Zur Kumpanei der MännerRECHTSbewegung. Münster: Unrast.

Kemper, Andreas. 2012. Die Maskulisten. Organisierter Antifeminismus im deutschsprachigen Raum. Münster: Unrast.

Keskinen, Suvi. 2013. Antifeminism and white identity politics. Nordic Journal of Migration Research 3:225-232.

Kinnvall, Catarina. 2004. Globalization and religious nationalism: self, identity, and the search for ontological security. Political Psychology 25:741-767.

Klaus, Elisabeth. 2008. Antifeminismus und Elitefeminismus - Eine Intervention. Feministische Studien 26:176-186.

Krause, Daniela, Beate Küpper, und Andreas Zick. 2015. Zwischen Wut und Druck: Rechtspopulistische Einstellungen in der Mitte. In Wut, Verachtung und Abwertung: Rechtspopulismus in Deutschland, Hrsg. Andreas Zick, Beate Küpper, 44-60. Bonn: Dietz.

Lang, Juliane, und Ulrich Peters. 2018. Antifeminismus in Deutschland. In Antifeminismus in Bewegung. Aktuelle Debatten um Geschlecht und sexuelle Vielfalt, Hrsg. Juliane Lang, Ulrich Peters, 13-36. Hamburg: Marta Press.

Lange, Robert V., und Marika Tiggemann. 1981. Dimensionality and reliability of the Rotter IE locus of control scale. Journal of Personality Assessment 45(4):398-406.

Laumann, Vivien, und Katharina Debus. 2018. „Frühsexualisierung“ und „Umerziehung“? Pädagogisches Handeln in Zeiten antifeministischer Organisationen und Stimmungsmache. In Antifeminismus in Bewegung. Aktuelle Debatten um Geschlecht und sexuelle Vielfalt, Hrsg. Juliane Lang, Ulrich Peters, 275-301. Hamburg: Marta Press.

List, Elisabeth. 1989. Denkverhältnisse. Feminismus als Kritik. In Denkverhältnisse. Feminismus und Kritik, Hrsg. Elisabeth List, Herlinde Studer, 7-37. Frankfurt am Main: Suhrkamp.

Mannheim, Karl. 1929. Ideologie und Utopie. Bonn: Friedrich Cohen.

Mannheim, Karl. 2002. Ideology and Utopia. An Introduction to the Sociology of Knowledge. London: Routledge \& Kegan Paul.

McAdam, Doug, Sidney Tarrow, und Charles Tilly. 2001. Dynamics of contention. Cambridge: Cambridge University Press.

Meyer, David S., und Suzanne Staggenborg. 1996. Movements, countermovements, and the structure of political opportunity. American Journal of Sociology 101:1628-1660.

Möller, Simon. 1999. Sexual Correctness: Die Modernisierung antifeministischer Debatten in den Medien. Opladen: Springer VS.

Mottl, Tahi L. 1980. The analysis of countermovements. Social Problems 27:620-635.

Nagoshi, Julie L., Katherine A. Adams, Heather K. Terrell, Eric D. Hill, Stephanie Brzuzy, und Craig T. Nagoshi. 2008. Gender differences in correlates of homophobia and transphobia. Sex Roles 59(78):521-531. 
Näser-Lather, Marion, Anna Lena Oldemeier, und Dorothee Beck (Hrsg.). 2019. Backlash!? Antifeminismus in Wissenschaft, Politik und Gesellschaft. Königstein: Helmer.

Nave-Herz, Rosemarie. 1997. Die Geschichte der Frauenbewegung in Deutschland. Bonn: Bundeszentrale für politische Bildung.

Peetz, Katharina. 2014. Zur Verflechtung von Antisemitismus, Antifeminismus und Emanzipation im Kaiserreich. Theologie. Geschichte Beihefte 8:121-256.

Petchesky, Rosalind P. 1981. Antiabortion, antifeminism, and the rise of the new right. Feminist Studies 7:206-246.

Pickel, Gert, und Anja Gladkich. 2012. Religious social capital in Europe. Connections between religiosity and civil society. In Transformations of religiosity-religion and religiosity in central and eastern Europe 1989-2010, Hrsg. Gert Pickel, Kornelia Sammet, 69-94. Wiesbaden: Springer VS.

Planert, Ute. 1998. Antifeminismus im Kaiserreich: Diskurs, soziale Formation und politische Mentalität. Göttingen: Vandenhoeck \& Ruprecht.

Pratto, Felicia, James Sidanius, Lisa M. Stallworth, und Bertram F. Malle. 1994. Social dominance orientation: a personality variable predicting social and political attitudes. Journal of Personality and Social Psychology 67(4):741-763.

Pusch, Luise F. 1983. Zur Einleitung: Feminismus und Frauenbewegung - Versuch einer Begriffskläung. In Feminismus. Inspektion der Herrenkultur, Hrsg. Luise F. Pusch, 9-18. Frankfurt am Main: Suhrkamp.

Putnam, Robert D. 2000. Bowling alone. The collapse and revival of American community. New York: Simon \& Schuster.

Radcliffe Richards, J. 1983. Welche Ziele der Frauenbewegung sind feministisch? In Feminismus. Inspektion der Herrenkultur, Hrsg. Luise F. Pusch, 18-33. Frankfurt am Main: Suhrkamp.

Reich, John, und Frank Infurna. 2017. Perceived control. Theory, research, and practice in the first 50 years. New York: Oxford University Press.

Reuband, Karl H. 2017. Die Dynamik des Pegida Protests. Der Einfluss von Ereignissen und bewegungsspezifischer Mobilisierung auf Teilnehmerzahlen und Teilnehmerzusammensetzung. Mitteilungen des Instituts für Deutsches und Internationales Parteienrecht und Parteienforschung 23:112-130.

Rosenbrock, Hinrich. 2012. Die antifeministische Männerrechtsbewegung. Denkweisen, Netzwerke und Online-Mobilisierung. Berlin: Heinrich-Böll-Stiftung.

Rotter, Julian B. 1966. Generalized expectancies of internal versus external control of reinforcements. Psychological Monographs 80:1-28.

Rucht, Dieter. 1991. Das Kräftefeld soziale Bewegungen, Gegenbewegungen und Staat: Einführende Bemerkungen. Forschungsjournal Soziale Bewegungen 4:9-17.

Schenk, Herrad. 1981. Die feministische Herausforderung. München: Beck.

Schmincke, Imke. 2018. Frauenfeindlich, sexistisch, antifeministisch? Begriffe und Phänomene bis zum aktuellen Antigenderismus. Aus Politik und Zeitgeschichte 68(17):28-33.

Schmincke, Imke. 2019. Familienbilder in Diskursen des Rechtspopulismus. In Komplexe Dynamiken globaler und lokaler Entwicklungen Verhandlungen des 39. Kongresses der Deutschen Gesellschaft für Soziologie in Göttingen 2018, Bd. 39, Hrsg. Nicole Burzan, 39-44.

Schütz, Alfred, und Thomas Luckmann. 2003. Die Lebenswelt des Alltags. Konstanz: UKV.

Schützeichel, Rainer. 2012. Wissenssoziologie. In Handbuch der Wissenssoziologie, Hrsg. Sabine Maasen, Mario Kaiser, und Martin Reinhart, 17-27. Wiesbaden: Springer.

Srole, Leo. 1956. Social integration and certain corollaries. An exploratory study. American Sociological Review 21:709-716.

Stögner, Karin. 2017. Angst vor dem ,,neuen Menschen“. Zur Verschränkung von Antisemitismus, Antifeminismus und Nationalismus in der FPÖ. In AfD \& FPÖ: Antisemitismus, völkischer Nationalismus und Geschlechterbilder, Hrsg. Stephan Grigat, 137-162. Baden-Baden: Nomos.

Thomas, William, und Dorothy Thomas. 1928. The definition of the situation. In Symbolische Interaktion. Arbeiten zu einer reflexiven Soziologie, Hrsg. Heinz Steinert, 33-335. Stuttgart: Klett.

Tilly, Charles. 1976. The Vendée. Cambridge: Harvard University Press.

Volkov, Shulamit. 1978. Antisemitism as a cultural code: reflections on the history and historiography of antisemitism in imperial Germany. The Leo Baeck Institute Year Book 23:25-46.

Williams, Cristan. 2016. Radical inclusion: recounting the trans inclusive history of radical feminism. Transgender Studies Quarterly 3(1-2):254-258.

Wimbauer, Christiane, Mona Motakef, und Julia Teschlade. 2015. Prekäre Selbstverständnisse. Anti-Genderismus: Sexualität und Geschlecht als Schauplätze aktueller politischer Auseinandersetzungen. In Anti-Genderismus, Hrsg. Sabine Hark, Paula-Irene Villa, 41-59. Bielefeld: transcript. 
Annette Schnabel ist Professorin für Soziologie und Soziologische Theorie an der Heinrich-Heine-Universität Düsseldorf. Wissenschaftliche Interessen sind: Religiöse und nationale Identitäten, Geschlecht, Wohlfahrtsstaat und soziale Bewegungen insbesondere aus der Perspektive der Theorien rationaler Handlungswahl. Adressiert werden diese Themen sowohl theoretisch als auch empirisch-quantitativ; seit 2017 Mitherausgeberin der ZRGP (Zeitschrift für Religion, Gesellschaft und Politik) und der ZfS (Zeitschrift für Soziologie). Aktuelle Publikationen sind: Religion und ihre Einbettung in Verfassungen als Kontext. ZRGP 2021 (zusammen mit Lisa Höhnes); The cultural code of antifeminist communication: Voicing opposition to the „Feminist Zeitgeist“. Acta Sociologica 2018 (zusammen mit H. Beyer und M. Lach); Theorien sozialer Bewegung. Frankfurt am Main: Campus-Verlag (2017, zusammen mit H. Beyer).

Heiko Beyer ist Heisenberg-Professor für Soziologie an der Heinrich-Heine-Universität Düsseldorf und unter anderem Chefredakteur der Zeitschrift für Religion, Gesellschaft und Politik (ZRGP). Seine Forschungsinteressen umfassen neben der Religionssoziologie die Themen Antisemitismus, Antifeminismus und Antiamerikanismus sowie die Bereiche der Soziologie der Menschenrechte, der Ideologiekritik, der Forschung zu Sozialen Bewegungen und zur soziologischen Theoriegeschichte. Zu seinen jüngsten Veröffentlichungen zählen die Monografie Horizont der Moderne. Zur Rolle der Amerikanischen Revolution in der Frühgeschichte der Soziologie. Frankfurt am Main: Campus Verlag (2021); der Sammelband Die Rückkehr der Ideologie. Zur Gegenwart eines Schlüsselbegriffs. Frankfurt am Main: Campus Verlag (2021, mit Alexandra Schauer) sowie beispielsweise die Zeitschriftenartikel Examining Discrimination in Everyday Life: A Stated Choice Experiment on Racism in the Sharing Economy. Journal of Ethnic and Migration Studies 2021 (mit Ulf Liebe) und The cultural code of antifeminist communication: Voicing opposition to the „Feminist Zeitgeist“. Acta Sociologica 2018 (mit A. Schnabel und M. Lach).

Bettina Ülpenich ist wissenschaftliche Mitarbeiterin am Lehrstuhl für Soziologie und soziologische Theorie an der Heinrich-Heine-Universität Düsseldorf. Im Jahr 2020 hat sie ihre Promotion unter dem Titel „Die Konstruktion kategorialer Zugehörigkeit am Beispiel von Alterszuschreibungen“ erfolgreich abgeschlossen. Zu ihren wissenschaftlichen Interessen zählen: Soziale Kategorisierung und Differenzkonstruktionen, Migrations- und Geschlechterforschung, die sie aus einer qualitativen Forschungsperspektive in den Blick nimmt. 